\title{
Ground-state phase diagram of the one-dimensional $t$ - $J$ model with pair hopping terms
}

\author{
J. R. Coulthard, ${ }^{1}$ S. R. Clark, ${ }^{2,3}$ and D. Jaksch ${ }^{1,4}$ \\ ${ }^{1}$ Clarendon Laboratory, University of Oxford, Parks Road, Oxford OX1 3PU, United Kingdom \\ ${ }^{2}$ Department of Physics, University of Bath, Claverton Down, Bath BA2 7AY, United Kingdom \\ ${ }^{3}$ Max Planck Institute for the Structure and Dynamics of Matter, University of Hamburg CFEL, Hamburg 22761, Germany \\ ${ }^{4}$ Centre for Quantum Technologies, National University of Singapore, 3 Science Drive 2, Singapore 117543
}

(Received 27 April 2018; revised manuscript received 28 June 2018; published 13 July 2018)

\begin{abstract}
The $t-J$ model is a standard model of strongly correlated electrons, often studied in the context of high- $T_{c}$ superconductivity. However, most studies of this model neglect three-site terms, which appear at the same order as the superexchange $J$. As these terms correspond to pair hopping, they are expected to play an important role in the physics of superconductivity when doped sufficiently far from half filling. We present a density matrix renormalization-group study of the one-dimensional $t-J$ model with the pair hopping terms included. We demonstrate that these additional terms radically change the one-dimensional ground-state phase diagram, extending the superconducting region at low fillings, while at larger fillings, superconductivity is completely suppressed. We explain this effect by introducing a simplified effective model of repulsive hardcore bosons.
\end{abstract}

DOI: 10.1103/PhysRevB.98.035116

\section{INTRODUCTION}

The $t-J$ model has long been a subject of intense interest as a prototypical model of strongly correlated electrons because it encapsulates the physics of constrained hopping and magnetically induced real-space pairing. As such, the $t-J$ model has been widely studied for its relevance to high- $T_{c}$ superconductivity $[1,2]$, in particular with connection to resonating valence bond (RVB) physics [3,4], and as a microscopic origin for the $S O(5)$ model of antiferromagnetism and superconductivity [5]. Traditionally, the $t-J$ model emerges as an effective low-energy description of the paradigmatic Hubbard model in the limit $t \ll U$ to second order in $t / U$, where $U$ is the on-site Coulomb repulsion, giving rise to a superexchange $J=4 t^{2} / U[6,7]$. The validity of the $t-J$ model in this context therefore necessitates $J / t \ll 1$. For high- $T_{c}$ superconductors, the regime of interest is $J \sim 0.3 t$ for a two-dimensional (2D) square lattice system close to half filling.

Despite its long history, there is increasing motivation to reexamine the $t-J$ model and explore its properties over a wider parameter space. A prominent case for this comes from the recent advances in generating strong $\mathrm{THz}$ fields in pump-probe experiments on solids. This technique now makes it possible to transiently manipulate materials by exciting them into nonequilibrium states not accessible thermally [8-11]. Such strongly driven systems are often described by effective Hamiltonians with significant differences from those in equilibrium [12-16]. In particular, the $t-J$ model originating from a periodically driven Hubbard model breaks the perturbative connection between $t$ and $J$, allowing $J / t$ to be controlled and the physics with $J / t>1$ to be probed [17]. Complementary to solid-state systems, the direct implementation of the Hubbard model and an experimental resolution of its low-temperature phase diagram is a longstanding goal of experiments with ultracold fermionic quantum gases in optical lattices $[18,19]$. In these synthetic solids, strong periodic driving, such as lattice shaking, is also routinely used to engineer the band structure [20] and microscopic interactions of the system [14], as demonstrated recently for the superexchange [21,22]. Thus, mapping out the complete phase diagram of the $t-J$ model provides a fuller picture of the strongly correlated states one might engineer by driving the Hubbard model.

Motivated by these developments, in this paper we examine the ground states of the $t-J$ model in $1 \mathrm{D}$ over a wide range of $J / t$ and fillings. While much of the focus on the $t-J$ model is in $2 \mathrm{D}$ systems, the $1 \mathrm{D}$ system nonetheless possesses a rich phase diagram. Indeed, it displays insulating, spin-gapped, and superconducting phases similar to the phenomenology of correlated materials in higher dimensions. Moreover, in 1D, the density matrix renormalization group (DMRG) $[24,25]$ provides an unprecedented ability to diagnose these exotic phases in an unbiased way for large systems, allowing for accurate extrapolation to the thermodynamic limit.

To correctly capture all the physics arising from the $t-J$ model with varied fillings, we crucially retain the singletpair-hopping term. Formally, this three-site term arises from the Hubbard model to the same order as the superexchange $J$. Close to half filling, it is often argued that pair hopping processes are rare [7], and so most previous studies of the $t-J$ model have neglected this term [23,26-28]. However, previous work on the large- $U$ Hubbard model has demonstrated that pair hopping is essential to understand its optical properties when doped away from half filling [29,30]. Some earlier works analyzing phases of the $t-J$ model have taken pair hopping into account [31-35], but were restricted to mean-field approximations or used exact diagonalizations on very small systems. A key contribution of our work is that we address the $t-J$ model without these limitations. We find that the inclusion of pair hopping leads to a dramatically different groundstate phase diagram. In particular, it has a significant impact on superconductivity by pushing the metal-superconducting boundary to lower values of $J / t$ at dilute fillings. Closer 
to half filling, the pair hopping simultaneously increases the size of the spin-gapped region and leads to the suppression of superconductivity, in line with mean-field calculations in two dimensions [35]. We explain this effect by considering a simplified model of constrained hardcore bosons.

The structure of this paper is as follows. In Sec. II we introduce the $t$ - $J$ model and discuss the pair hopping term. In Sec. III we present a selection of phase diagrams and discuss how we characterize the various phases. We then introduce in Sec. IV a constrained hardcore boson model and compare its properties to those of the $t-J$ model. Finally, we conclude in Sec. V.

\section{THE $t$ - $J$ MODEL}

In the limit $t \ll U$ of the Hubbard model, double occupancies are energetically suppressed. However, second-order processes, where different singly occupied configurations are connected by virtual excitations to and from these doubly occupied states, give rise to the $t$ - $J$ model describing the effective low-energy dynamics. The $t$ - $J$ model Hamiltonian may be written formally as [31]

$$
\begin{aligned}
\hat{H}_{t J \alpha}= & -t \sum_{\langle i j\rangle \sigma}\left(\hat{f}_{i, \sigma}^{\dagger} \hat{f}_{j, \sigma}+\text { H.c. }\right) \\
& -J \sum_{\langle i j\rangle} \hat{b}_{i j}^{\dagger} \hat{b}_{i j}-\alpha J \sum_{\langle i j k\rangle}\left(\hat{b}_{i j}^{\dagger} \hat{b}_{j k}+\text { H.c. }\right),
\end{aligned}
$$

where $t$ is the single-particle hopping amplitude, $J=4 t^{2} / U$ is the strength of the superexchange interaction, and $\alpha$ is a dimensionless constant of order unity. The definition of this model is built from projected fermionic annihilation operators for a spin$\sigma$ fermion on lattice site $j$, defined as $\hat{f}_{j, \sigma}=\hat{c}_{j, \sigma} \hat{P}$, where $\hat{c}_{j, \sigma}$ is the corresponding canonical fermionic annihilation operator. Here, $\hat{P}$ is a projector that implements the exclusion of double occupations and is given by $\hat{P}=\prod_{j}\left(1-\hat{n}_{j, \uparrow} \hat{n}_{j, \downarrow}\right)$, where $\hat{n}_{j, \sigma}=\hat{c}_{\sigma, j}^{\dagger} \hat{c}_{\sigma, j}$ is the number operator for spin- $\sigma$ fermions on site $j$. The operator

$$
\hat{b}_{i, j}=\frac{1}{\sqrt{2}}\left(\hat{f}_{i, \downarrow} \hat{f}_{j, \uparrow}-\hat{f}_{i, \uparrow} \hat{f}_{j, \downarrow}\right)
$$

annihilates a spin singlet on lattice sites $i$ and $j$.

The $t$ - $J$ model captures two significant pieces of physics. First, it subjects the motion of electrons in a tight-binding band with hopping amplitude $t$ to a local constraint that excludes double occupancies. Specifically, unlike $\hat{c}_{j, \sigma}$, the projection means that $\hat{f}_{j, \sigma}$ operators do not obey the canonical fermionic anticommutation relations. This induces a nonFermi-liquid metallic state and accounts for density-dependent band-narrowing effects [6]. Second, neighboring electrons experience an antiferromagnetic Heisenberg superexchange with amplitude $J$. This induces real-space singlet pairing of electrons, which can subsequently hop with amplitude $\alpha J$, and accounts for the formation superconducting and magnetically ordered insulating states.

The parameter $\alpha$ is equal to $1 / 2$ for a $t-J$ model arising from the equilibrium Hubbard model. To distinguish the Hamiltonian in Eq. (1) from the typically studied $t-J$ model, which takes $\alpha=0$, we refer to it as the $t-J-\alpha$ model from now on. Motivated by the effects of strong periodic driving on the Hubbard model, we consider the regime $0 \leqslant J / t \leqslant 8$ and $0 \leqslant \alpha \leqslant 1 / 2$ not accessible from equilibrium. Specifically, in Appendix A we show how periodic driving can be used to control both the single-particle hopping and the pair hopping terms, while leaving the superexchange unchanged.

\section{PHASE DIAGRAM}

To compute the ground-state phase diagram of the $t-J-\alpha$ model, we use the finite system DMRG algorithm [24,25] as implemented in the open-source Tensor Network Theory (TNT) library [36]. Further details of the DMRG calculation are provided in Appendix B. We consider a 1D chain of $L$ sites containing a number of "up" and "down" fermions $N_{\uparrow}$ and $N_{\downarrow}$, respectively, where $N_{\sigma}=\left\langle\sum_{j} \hat{n}_{j, \sigma}\right\rangle$, and $\langle\cdot\rangle$ denotes the expectation value with respect to the ground state. Fixing this filling results in a mean number of fermions per site, $n=\left(N_{\uparrow}+N_{\downarrow}\right) / L$. Note that except when determining the spin gap in Sec. III B, we take $N_{\uparrow}=N_{\downarrow}$.

The main correlation functions of interest are the densitydensity correlations

$$
N_{i j}=\left\langle\hat{n}_{i} \hat{n}_{j}\right\rangle-\left\langle\hat{n}_{i}\right\rangle\left\langle\hat{n}_{j}\right\rangle
$$

with $\hat{n}_{j}=\hat{n}_{j \uparrow}+\hat{n}_{j \downarrow}$, the spin-spin correlations

$$
S_{i j}=\left\langle\hat{S}_{i}^{z} \hat{S}_{j}^{z}\right\rangle
$$

with $\hat{S}_{i}^{z}=\left(\hat{n}_{i, \uparrow}-\hat{n}_{i, \downarrow}\right) / 2$, and the nearest-neighbor singletpairing correlations

$$
P_{i j}=\left\langle\hat{b}_{i, i+1}^{\dagger} \hat{b}_{j, j+1}\right\rangle .
$$

We also compute the corresponding structure factors, i.e., the Fourier transforms of these quantities,

$$
X(q)=\frac{1}{L} \sum_{j k} X_{j k} e^{i q(j-k)},
$$

where $X$ is any of $N, S$, or $P$.

Our main results, i.e., the phase diagrams for the $t-J-\alpha$ model in the $n-J / t$ plane, are presented in Fig. 1 . For $\alpha=0$, we reproduce the results of Moreno et al. [23]. We also show the phase diagrams for $\alpha=0.15$ and $\alpha=1 / 2$, respectively, mapping the full range of phases induced by the pair hopping.

For all three values of $\alpha$ shown in Fig. 1, the ground state for small $J / t$ is a correlated metal. As the strength of the superexchange interaction increases, antiferromagnetic pairs begin to form and the system crosses into a superconducting phase (SC), which exhibits a slow algebraic decay of singlet correlations, as we discuss in Sec. IIIB. Further information about the nature of pairs in the system is provided by the presence or absence of a spin gap (SG). As we discuss in Sec. IV, the presence of a finite spin gap indicates that all fermions in the system are bound into nearest-neighbor singlet pairs. Contrary to the small exact diagonalization results of Ammon et al. [32], we find that superconductivity does not survive at all filling fractions. We do, however, find that the spin gap extends outside of the superconducting region, indicating a gas of preformed pairs.

For $\alpha=0$ and $\alpha=0.15$, at sufficiently large $J / t$, the superexchange interaction $J$ wins over kinetic energy $t$ and $\alpha J$, and the fermions and holes localize into separate clusters, 

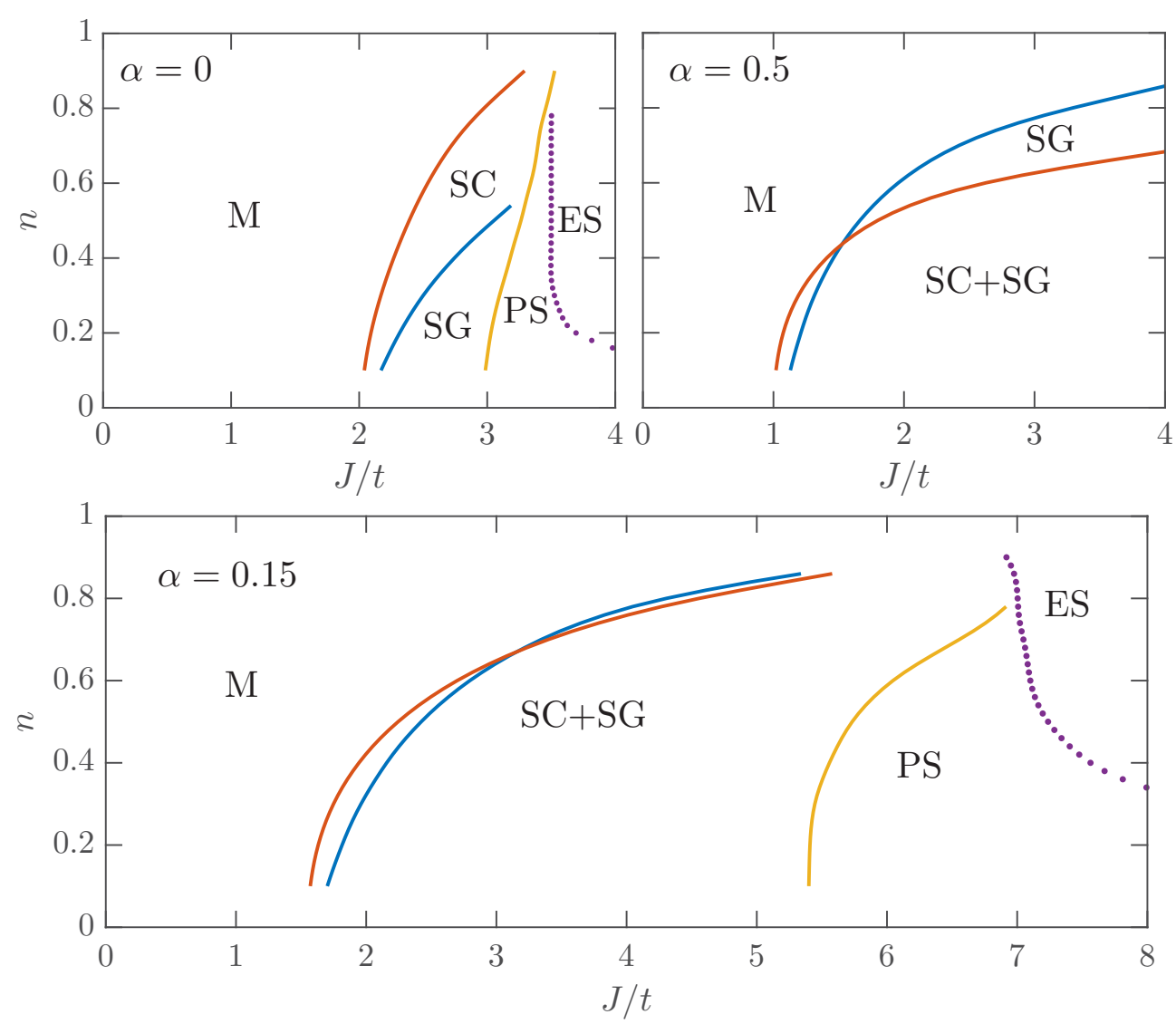

FIG. 1. Constant $\alpha$ slices through the $t-J$ - $\alpha$ phase diagram as a function of $J / t$ and $n$. Metallic (M), superconducting (SC), spin-gapped (SG), phase-separated (PS), and electron solid (ES) phases are marked. In the $\alpha=0.5$ case, the phase-separated and electron solid phases have vanished, which we have verified for up to $J / t=100$. The phase diagram for $\alpha=0$ is consistent with Moreno et al. [23].

known as phase separation (PS). This is signaled by a diverging compressibility, which we discuss in Sec. III A. As $J / t$ is increased further, we reach a regime where the maximum local density $\max _{j}\left\langle\hat{n}_{j}\right\rangle$ approaches unity, called the electron solid (ES) phase.

\section{A. Phase separation}

The second term in Eq. (1) is commonly rewritten as the antiferromagnetic Heisenberg coupling,

$$
\hat{H}_{\text {Heis }}=\frac{J}{2} \sum_{\langle i j\rangle}\left(\hat{S}_{i}^{x} \hat{S}_{j}^{x}+\hat{S}_{i}^{y} \hat{S}_{j}^{y}+\hat{S}_{i}^{z} \hat{S}_{j}^{z}-\frac{\hat{n}_{i} \hat{n}_{j}}{4}\right),
$$

where $\hat{S}_{j}^{x}=\left(\hat{f}_{j, \downarrow}^{\dagger} \hat{f}_{j, \uparrow}+\hat{f}_{j, \uparrow}^{\dagger} \hat{f}_{j, \downarrow}\right), \hat{S}_{j}^{y}=\mathrm{i}\left(\hat{f}_{j, \downarrow}^{\dagger} \hat{f}_{j, \uparrow}-\hat{f}_{j, \uparrow}^{\dagger} \hat{f}_{j, \downarrow}\right)$, and $\hat{S}_{j}^{z}=\left(\hat{n}_{j, \uparrow}-\hat{n}_{j, \downarrow}\right)$ are the spin-1/2 Pauli operators acting on the spin degree of freedom at site $j$. When written in this form, we anticipate that in the absence of pair hopping, the $t-J$ model will exhibit competition between the delocalizing effect of the single-particle hopping $t$ and the attractive Heisenberg-like interaction $J$. When $t \ll J$, we expect this attractive interaction to dominate and the fermions to separate into antiferromagnetic clusters and hole-rich regions. This is known as phase separation [37]. To quantitatively characterize the transition boundary, we compute the inverse compressibility,

$$
\begin{aligned}
\kappa^{-1}(n) & =n^{2} \frac{\partial^{2} E_{0}(n)}{\partial n^{2}} \\
& \approx n^{2} \frac{\left[E_{0}(n+\Delta n)+E_{0}(n-\Delta n)-2 E_{0}(n)\right]}{(\Delta n)^{2}},
\end{aligned}
$$

where $E_{0}(n)$ is the ground-state energy of the system at a filling $n$. At the onset of phase separation, the compressibility diverges and so $\kappa^{-1}$ crosses zero. The phase-separation boundary is shown in Fig. 2(a) for selected values of $\alpha$. We see clearly that the phase separation is suppressed with increasing $\alpha$. We further find that phase separation disappears completely for $\alpha=1 / 2$ [32].

Where phase separation does occur and if $J / t$ is sufficiently large, the system can become fully separated into an particlerich region with $\left\langle\hat{n}_{j}\right\rangle \approx 1$ and a hole-rich region with $\left\langle\hat{n}_{j}\right\rangle \approx 0$. This is illustrated in Fig. 2(b) for $\alpha=0$. In this plot, $J / t=2$ is metallic, while the rest are phase separated, and $J / t=3.5$ indicates an electron solid phase with regions of $\left\langle\hat{n}_{i}\right\rangle=1$ and $\left\langle\hat{n}_{i}\right\rangle=0$.

As noted in Ref. [23], we find that the phase-separated phase presents a number of issues for the DMRG calculation. First, the antiferromagnetic island is off-center for larger $J / t$. This is because the phase-separated ground state is highly degenerate, i.e., ignoring boundary effects, the cluster of fermions has very nearly the same energy regardless of where it is located in the 

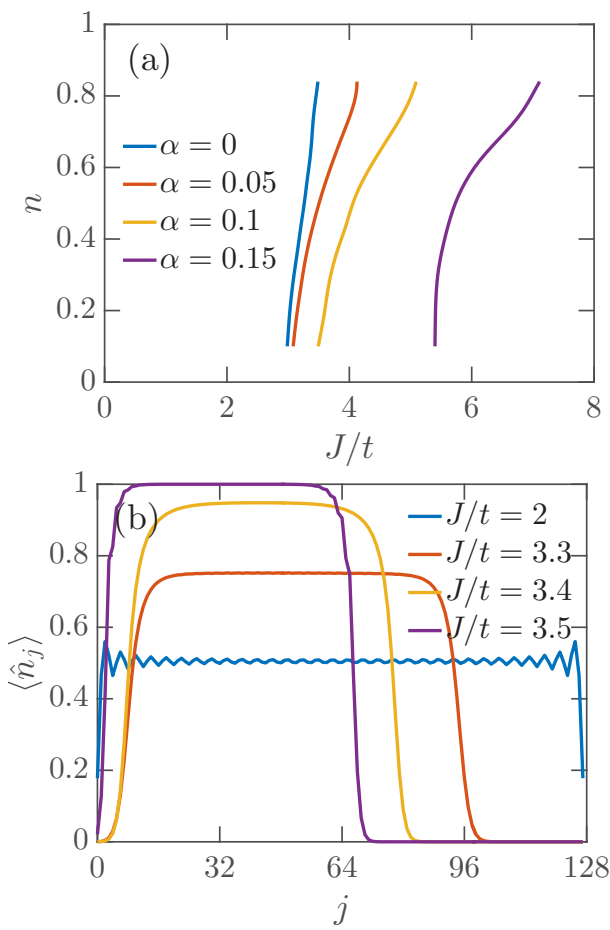

FIG. 2. (a) Phase diagram plot of $J$ vs $n$ indicating the phaseseparation boundary at various values of $\alpha$. (b) Examples of the ground-state real-space fermion density $\left\langle\hat{n}_{i}\right\rangle$ for $\alpha=0, n=0.5$ on $L=128$ sites, at various values of $J / t$.

lattice [38] Relatedly, we find that for large systems, DMRG encounters metastability issues deep in the phase-separated phase. The results for $J / t=3.5$ in Fig. 2(b) are therefore not expected to be quantitatively representative of the true ground state (as can be seen by the lack of reflection symmetry in the antiferromagnetic cluster). Because of this, a full extrapolation of the electron solid phase boundary to the thermodynamic limit is not possible. Rather, in Fig. 1, we show the approximate boundary as the contour where $\max _{j}\left\langle\hat{n}_{j}\right\rangle>0.999$ for $L=128$ as a dotted line.

\section{B. Superconducting region and spin gap}

To identify the superconducting phase boundary, we appeal to the Luttinger liquid formalism. When the $t-J-\alpha$ model is not phase separated, it can be mapped onto either a Tomonaga-Luttinger liquid (TLL) with gapless spin and charge excitations, or Luther-Emery liquid (LEL) with a spin gap $[23,39,40]$. The central quantity in both of these models is the Luttinger parameter $K_{\rho}$. For $K_{\rho}<1$, the TLL/LEL has repulsive interactions, whereas for $K_{\rho}>1$, the TLL/LEL has attractive interactions, and thus superconducting correlations dominate.

We extract $K_{\rho}$ by computing the ground-state density structure factor and exploiting the linear dependence at small- $q$ values, which we show in Fig. 3(a). The linear dependence is given by [23,39]

$$
N(q) \approx \frac{K_{\rho}|q|}{\pi} \text { as } q \rightarrow 0,
$$

for both the TLL and LEL. By performing a linear fit for small values of $q$, we obtain a value of $K_{\rho}$ at a given system size $L$. By computing this as a function of $L$, extrapolating to the $L \rightarrow \infty$ limit, and finding where $K_{\rho}=1$, we determine the superconducting phase boundary, which we show in Fig. 3(b) for a few values of $\alpha$. We clearly see that the effect of the pair hopping is to shift the metal-superconducting phase boundary to lower values of $J / t$ at small fillings and suppress SC at large fillings. We expect this observation to persist in higher dimensions, and indeed this is corroborated by two-dimensional renormalized mean-field theory studies [35]. In Fig. 3(c), we show some examples of the real-space singlet correlations $P_{0, r}$ at low fermion densities. Between $J / t=0.8$ and $J / t=0.5$, as the system enters the spin-gapped region, we see a clear change in behavior as $P_{0, r}$ goes from oscillatory and rapid (but still algebraic) decay to a much slower decaying behavior with suppressed oscillations. The changes are indicative of a gapless metal to spin-gapped metal to spin-gapped superconductor transition.

The spin gap is defined as the energy gap between the "singlet" ground state and the lowest-lying triplet excitation,

$$
E_{\mathrm{SG}}=E_{0}\left(S^{z}=1\right)-E_{0}\left(S^{z}=0\right),
$$

where $S^{z}=\left(N_{\uparrow}-N_{\downarrow}\right) / 2$. In any finite system, $E_{\mathrm{SG}}$ will be finite, vanishing only in the thermodynamic limit. It also closes rather slowly as a function of system size, and so it is again important to extrapolate to $L \rightarrow \infty$ [23]. The contours drawn in Fig. 1 are for $E_{\mathrm{SG}}(L \rightarrow \infty)<0.005$.

As we shall discuss in more detail in Sec. IV, the presence of a finite spin gap alters the nature of the superconducting ground state and it becomes possible to think of the superconductor as a Bose condensate of locally bound singlet pairs. Luttinger liquid theory predicts that the long-range behavior of the singlet correlations will be

$$
P_{0, r} \sim r^{-\left(1+1 / K_{\rho}\right)} \quad \text { and } \quad P_{0, r} \sim r^{-1 / K_{\rho}},
$$

in the TLL and LEL, respectively [40]. For reference, we indicate two algebraic decays in Fig. 3(c). The lower line is given by $0.16 r^{-1.25}$, while the upper line, given by $0.19 r^{-1}$, indicates superconducting correlations. The real-space singlet correlations for $J / t=2.2$ and $J / t=3.5$ are therefore consistent with a superconducting LEL with $K_{\rho} \gtrsim 1$.

\section{Magnetic correlations}

From the Heisenberg term given by Eq. (3), it is clear that the superexchange interaction will induce antiferromagnetic correlations in the ground state. At precisely half filling, i.e., $n=1$, the fermions become completely immobile and we are left only with the spin degree of freedom, which is governed by the Heisenberg Hamiltonian with an antiferromagnetic ground state [7].

Away from half filling, the presence of holes obscures the underlying magnetic order as the single particle and pair hopping delocalize the spins. Rather than antiferromagnetic correlations (i.e., a spin wave with quasimomentum $q=\pi$ ), the ground state contains a spin wave with longer wavelength $q=n \pi$. This is identified by the location of the peak in the spin structure factor, which is shown in Fig. 4(a) for various fillings. In Fig. 4(b), we show the spin structure 

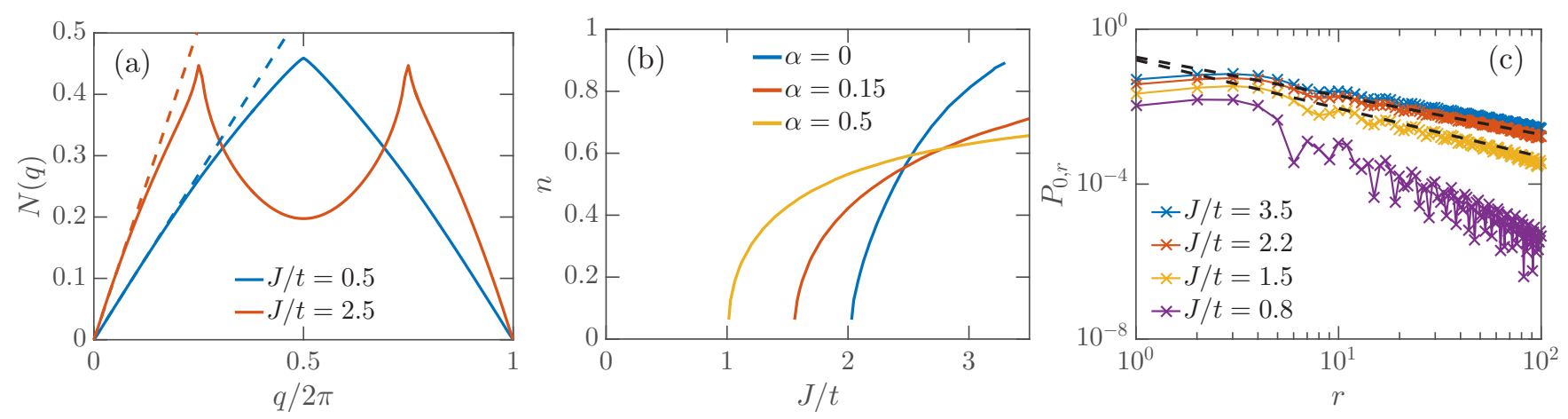

FIG. 3. (a) The density structure factor $N(q)$ for $\alpha=0.5, L=128$ sites. The dotted lines show the linear fit as $q \rightarrow 0$, from which $K_{\rho}$ is extracted. (b) $K_{\rho}=1$ contours for $\alpha=0,0.15$, and 0.5 , extrapolated to the thermodynamic limit. (c) The real-space singlet correlations $P_{0, r}$ are marked with crosses, with a solid line as a guide for the eye. Computed for $L=128, \alpha=0.5, n=0.5$, at various values of $J / t$. The upper and lower black dotted lines indicate the power-law decays $0.19 r^{-1}$ and $0.16 r^{-1.25}$, respectively.

factor at various $J / t$. Upon entering the spin gap, the sharp peaks, which suggest quasi-long-range magnetic order, are suppressed and are instead replaced by a broad peak at $q=\pi$. This is readily understood by considering the spin-gapped phase as a gas of bound singlet pairs, which we will discuss in more detail in Sec. IV. Each singlet pair's spin degree of freedom is maximally entangled and so, due to the monogamy of entanglement, the constituent fermions cannot have any spin correlations beyond their adjacent partner. Hence this peak has the approximate form $S(q) \sim 1-\cos (q)$, which is the form given by a free gas of antiferromagnetically bound pairs [32] and which we indicate as a black dotted line in Fig. 4(b).
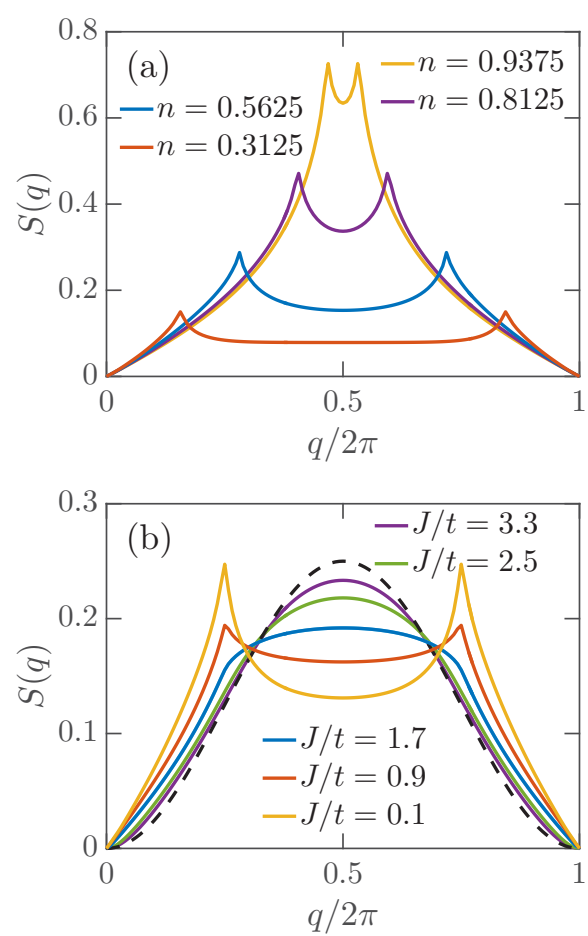

FIG. 4. Spin structure factor $S(q)$ for $L=128$ sites with $\alpha=0.5$. (a) $S(q)$ at $J / t=0.1$ at various fillings $n$; (b) $S(q)$ for $\alpha=1 / 2$ and $n=0.5$ at various values of $J / t$. For reference, the black dashed line indicates $(1 / 8)[1-\cos (q)]$, which is the structure factor for a free gas of bound pairs.

\section{EFFECTIVE BOSONIC MODEL}

We have so far demonstrated that, counterintuitively, a finite pair hopping $\alpha>0$ leads to suppressed superconductivity at large fillings, coinciding with an increased spin-gap region. To better understand this observation, we now look at the spin-gapped region in more detail. Inside this region, we expect all fermions to be bound into singlet pairs. Given this, we define a new Hilbert space for the system consisting of $L-1$ "sites," which represent the bonds of the original lattice, as illustrated in Fig. 5. These sites may (or may not) be occupied by a boson representing a singlet pair in the spin-gapped $t-J-\alpha$ model and thus we have $n L / 2$ bosons in the system. The operators $\hat{a}_{j}^{\dagger}$ and $\hat{a}_{j}$ create and annihilate hardcore bosons on site $j$, respectively, while $\hat{m}_{j}=\hat{a}_{j}^{\dagger} \hat{a}_{j}$ is the corresponding bosonic number operator.

However, not all configurations of this effective lattice model represent valid configurations in the $t-J-\alpha$ model. Since the $t-J-\alpha$ model does not allow double occupations, two nearest-neighbor singlet pairs cannot overlap. This manifests itself in the effective bosonic model as the constraint that we cannot have two adjacent sites occupied by hardcore bosons. This constraint is implemented via the projection operator $\hat{P}_{\mathrm{nn}}=\prod_{j}\left(1-\hat{m}_{j} \hat{m}_{j+1}\right)$, which kills any state containing bosons on adjacent sites. This constraint fortuitously prevents inconsistencies which would arise due to the singlet creation operators $b_{j, j+1}^{(\dagger)}$ not obeying bosonic commutation relations when the singlets overlap. Rather, states which

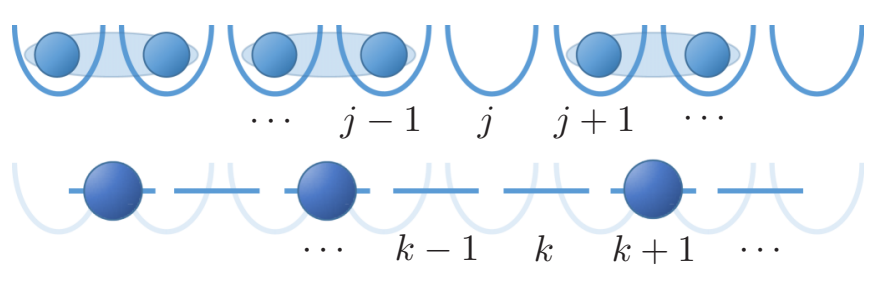

FIG. 5. Schematic showing how a singlet pair of fermions is represented in our effective model by a single hardcore boson. Due to the "no-double-occupancy" constraint in the $t$ - $J$ - $\alpha$ model, singlets cannot overlap, and so the hardcore bosons cannot occupy adjacent sites. 

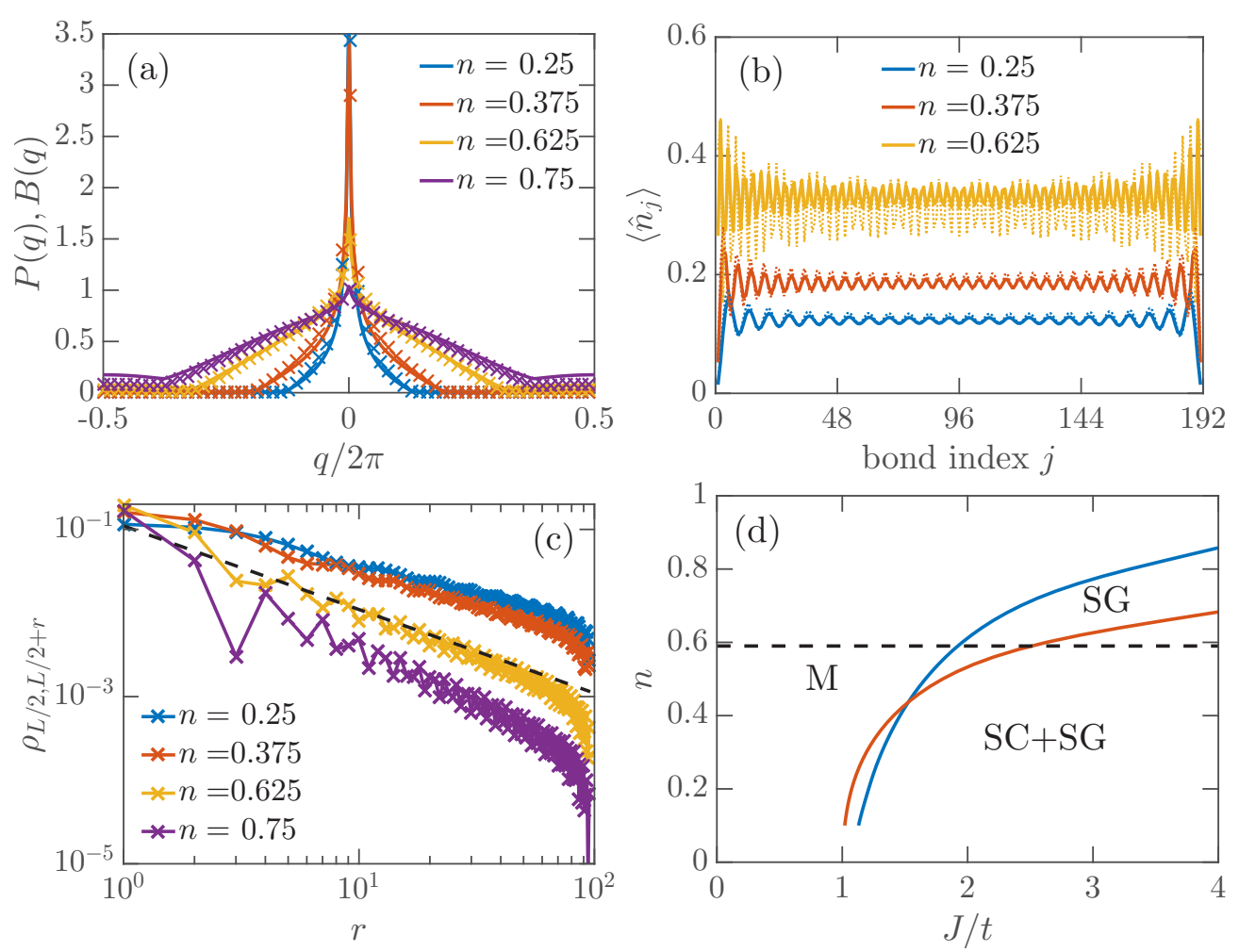

FIG. 6. A comparison between the $t-J$ - $\alpha$ model and the projected boson model. The $t-J$ - $\alpha$ model is with $J / t=5$, and $\alpha=1 / 2$ computed with $L=192$ lattice sties. (a) The singlet structure factor $P(q)$ is shown as the solid line, with the boson structure factor $B(q)$ shown with crosses. (b) A comparison of the real-space densities of singlets and bosons, with the singlets shown with the solid line and the bosons marked with the dotted line. (c) A slice of the single boson density matrix as a function of separation $r$ is marked with crosses, with solid lines as a guide to the eye. For reference, a black dashed line indicates the line $0.11 r^{-1}$. (d) A duplication of the $t-J-\alpha$ model phase diagram at $\alpha=0.5$ from Fig. 1, but now including the superfluid phase boundary of the effective boson model marked as a black dotted line.

would reveal the composite nature of the bosons are projected out. This effective boson model is closely related to quantum lattice dimer models [41] thought to have relevance to high- $T_{c}$ superconductivity in two dimensions. In the limit $J / t \rightarrow \infty$, the Hamiltonian for the effective model is

$$
\hat{H}_{\mathrm{eff}}=\hat{P}_{\mathrm{nn}}\left[-J \sum_{j} \hat{m}_{j}-\alpha J \sum_{j}\left(\hat{a}_{j}^{\dagger} \hat{a}_{j+1}+\text { H.c. }\right)\right] \hat{P}_{\mathrm{nn}} .
$$

This can be seen as a Hamiltonian for hardcore bosons hopping on a lattice with an infinite nearest-neighbor repulsion. Since the total number of bosons is conserved and we are working at a fixed filling fraction $n$, the first term is a constant $n L J / 2$, and thus can be ignored. The parameter $\alpha J$ then just rescales the energies and does not modify the ground state. This leaves the filling fraction $n$ as the only free parameter in the model. Despite the restriction $\hat{P}_{\mathrm{nn}}$ on the hopping, we still expect the bosons to be able to quasicondense into a superfluid state when the filling is sufficiently small, $n<n_{\text {crit }}$. This superfluid of bosons then corresponds to spin-gapped superconductivity in the $t-J-\alpha$ model.

The boson structure factor $B(q)$ is Eq. (2) applied to the single-particle density matrix (SPDM) $\rho_{j k}=\left\langle\hat{a}_{j}^{\dagger} \hat{a}_{k}\right\rangle$, and is essentially the momentum distribution of bosons. This is shown as crosses in Fig. 6(a), where we see that when the filling is small, the bosons do not see the extremely strong local repulsive interaction and so they macroscopically occupy the $q=0$ quasimomentum state. However, as the filling increases, the repulsive interaction plays a stronger role and the peak broadens as the bosons are forced, by the interactions, to occupy higher momentum states (quantum depletion of the quasicondensate). In the same figure, we compare this with the singlet structure factor of the $t-J-\alpha$ model with $J / t=5$ (solid lines), finding a very close agreement between the two.

Similarly, the real-space boson density is shown in Fig. 6(b). At smaller fillings, small oscillations at a frequency $\pi n$ can clearly be seen. This is because in one dimension, hardcore bosons inherit the Friedel oscillations from the corresponding Jordan-Wigner fermions [42]. Once again, these closely match the oscillations in the real-space singlet density in the $t-J-\alpha$ model ground state.

Whether a bosonic lattice system is superfluid or not is determined by the decay of off-diagonal elements in the SPDM. To determine the critical filling of the superfluid transition, we now look at the Luttinger parameter for bosons $K_{b}$ [43]. One can show that for $r \gg 1$, the long-range behavior of the SPDM is

$$
\rho_{0, r} \sim r^{-K_{b} / 2}
$$


which we show in Fig. 6(c). As in the fermionic case, we extract $K_{b}$ from the bosonic density structure factor,

$$
M(q) \approx \frac{|q|}{2 \pi K_{b}} \text { as } q \rightarrow 0,
$$

where $M(q)$ is Eq. (2) applied to the correlation function,

$$
M_{j, k}=\left\langle\hat{m}_{j} \hat{m}_{k}\right\rangle-\left\langle\hat{m}_{j}\right\rangle\left\langle\hat{m}_{k}\right\rangle .
$$

Computing $K_{b}$ as a function of $n$, we find that the critical filling $n_{\text {crit }} \approx 0.59$, which we mark on a copy of the $\alpha=$ $0.5 t-J-\alpha$ phase diagram, showing a qualitative agreement for the superconductor-preformed pair transition at large $J / t$.

In the vicinity of the $t-J-\alpha$ phase diagram where the effective boson model is valid (i.e., the region with a significant spin gap), this number provides an estimate of the largest filling at which one can have superconductivity. We expect the single-particle hopping $t$, which we have neglected in the effective boson model, to increase the propensity of the system to superconduct, and so $n_{\text {crit }}$ is expected to provide a lower bound on this maximum filling. We find that this number is in approximate agreement with the $t-J-\alpha$ model at $J / t \gg 1$, as we indicate in Fig. 6(d). Eventually, at maximum filling $n=1$, the ground state is a (pair) density wave with every other bond being occupied by a hardcore boson. The close agreement between these two models indicates that the effective model provides a quantitative description of pair condensation in the $t-J-\alpha$ model. The effective bosonic model makes it clear that the loss of superconductivity close to half filling is due to the kinetic constraints imposed by the nonoverlap of singlet pairs.

In addition, we find that DMRG calculations for the projected boson model converge much more rapidly than the $t-J-\alpha$ model, while accurately describing many of its properties. In $t-J-\alpha$ models where DMRG is slow to converge, it may be possible to accelerate these calculations in the spin-gapped regime by "coarse graining" to an effective bosonic model for the pairs, before interpolating to the full fermionic model [44].

\section{CONCLUSIONS}

We have shown that the effect of the pair hopping $\alpha J$ in the $t-J-\alpha$ model is to enhance the mobility of pairs, which manifests itself in the ground-state phase diagram in a number of ways. First, this pushes the metal-superconducting boundary to lower values of $J / t$ in dilute systems and destabilizes the phase-separated region. This has significant implications for periodically driven Hubbard systems, as it means that drivinginduced singlet pairing may be induced at significantly lower strengths than might be expected. Second, at larger fillings, superconductivity is suppressed despite the increased pair hopping. We now understand this in the following way: by lowering the energy of bound singlets, the pair hopping increases the size of the spin-gap region up to much larger values of $n$. Inside the spin-gap region, the physics may be described by a simple model of hardcore bosons with a kinetic constraint whose origins lie in the no-double-occupation projection of the $t-J$ model. Due to these restrictions, the bosons may not condense above a critical filling $n_{\text {crit }}$, and so superconductivity in the $t-J-\alpha$ model cannot occur inside the spin gap above this filling. This is consistent with the $\alpha=0.5$ phase diagram, which shows a larger spin-gapped region than superconducting region.

It is known that including next-nearest-neighbor hopping terms in one-dimensional chains (equivalent to a two-leg ladder system) can enhance the size of the spin-gap region [45]. This raises the intriguing possibility that kinetic constraints in the spin-gapped phase might play an important role in fully two-dimensional systems, and in particular of high- $T_{c}$ superconductors. It is likely that this behavior may be clearly observed in cold-atom experiments, where superexchange physics can be more directly probed [21,22]. In short, studying the $t-J-\alpha$ model in higher dimensions could provide significant insights into the behavior of high- $T_{c}$ superconductors and periodically driven strongly correlated systems.

\section{ACKNOWLEDGMENTS}

This research is funded by the European Research Council under the European Union's Seventh Framework Programme (FP7/2007-2013)/ERC Grant Agreement No. 319286 Q-MAC. D.J. acknowledges support from the EPSRC under Grants No. EP/K038311/1 and No. EP/P009565/1. S.R.C. gratefully acknowledges support from the EPSRC under Grant No. EP/P025110/1.

\section{APPENDIX A: FLOQUET ENGINEERING THE $t-J-\alpha$ MODEL}

Here we outline how one can engineer the $t-J-\alpha$ model out of equilibrium by periodically driving a Hubbard model. Possible experimental implementations include, for instance, shaking an optical lattice [21,22] or driving vibrations in an organic solid $[8,9,17]$. We begin with a one-dimensional singleband Hubbard model,

$$
\hat{H}_{\text {Hub }}=U \sum_{j} \hat{n}_{j, \uparrow} \hat{n}_{j, \downarrow}-t \sum_{j, \sigma}\left(\hat{c}_{j, \sigma}^{\dagger} \hat{c}_{j+1, \sigma}+\text { H.c. }\right) .
$$

We then add a periodic driving term,

$$
\hat{H}_{\text {drive }}(\tau)=V \sin (\Omega \tau) \sum_{j} j \hat{n}_{j} .
$$

This particular driving term models a cloud of ultracold atoms trapped in an optical lattice, where the lattice itself is shaken with an angular frequency $\Omega$, or an ac "electric field" is applied across the system. However, similar physics is shown to occur with other driving terms, such as those induced by a traveling wave [13], or where odd and even sites experience different driving strengths [17].

As the Hamiltonian $\hat{H}_{\text {Hub }}+\hat{H}_{\text {drive }}(\tau)$ is periodic in time, we are able to use Floquet theory [46-48] to compute an effective static Hamiltonian for the stroboscopic evolution of the system. In this instance, we focus on the far off-resonant, in-gap regime $t \ll \Omega \ll U$. We seek an effective Hamiltonian which describes the low-energy physics of the Hamiltonian, which we obtain via a generalized Schrieffer-Wolff transformation (SWT) [15]. The dynamics generated by the Hamiltonian will contain oscillations at frequencies $\Omega$ and $U$, both of which are large compared to $t$. The SWT amounts to a sequence of rotating wave approximations where we systematically eliminate frequencies from highest to lowest. 

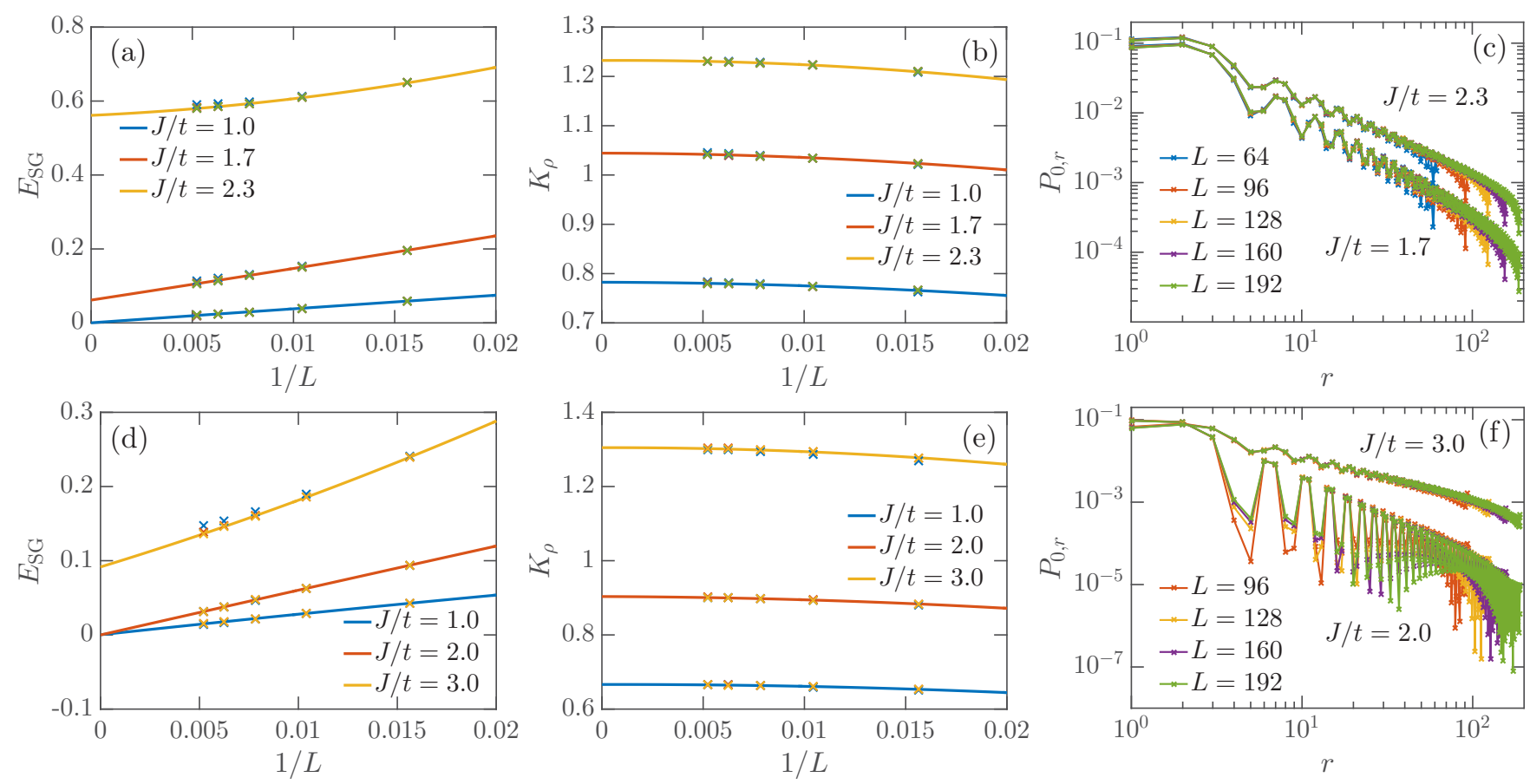

FIG. 7. Examples of the finite-size extrapolation; the system parameters are (a)-(c) $\alpha=0.5, n=7 / 32$, and (d)-(f) $\alpha=0.15, n=1 / 2$, with $J / t$ marked on the plots. (a),(d) The extrapolation of the spin gap; (b),(e) the Luttinger Parameter extrapolation. The crosses (which overlap) are computed for bond dimensions $\chi=100-500$. (c) The singlet correlations at selected interaction strengths with increasing system size computed for $\chi=300$.

We begin by performing the standard SWT to order $t / U$ to obtain the $t-J-\alpha$ model,

$$
\begin{aligned}
\hat{H}_{t J \Omega}= & \hat{P}\left[-t_{0} \sum_{j, \sigma}\left(c_{j, \sigma}^{\dagger} c_{j+1, \sigma}+\text { H.c. }\right)\right. \\
& -\frac{t_{0}^{2}}{U} \sum_{j} \hat{b}_{j, j+1}^{\dagger} \hat{b}_{j, j+1}+\frac{t_{0}^{2}}{2 U}\left(\hat{b}_{j, j+1}^{\dagger} \hat{b}_{j+1, j+2}+\text { H.c. }\right) \\
& \left.+V \cos (\Omega \tau) \sum_{j} j \hat{n}_{j}\right] \hat{P},
\end{aligned}
$$

where $\hat{P}$ is a projector onto the state which contain no double occupations. From here, we transform into the rotating frame with respect to the driving term and perform a high-frequency Magnus expansion [13] to obtain the effective Hamiltonian,

$$
\begin{aligned}
\hat{H}_{t J \alpha}= & \hat{P}\left[-\mathcal{J}_{0}\left(\frac{V}{\Omega}\right) t_{0} \sum_{j, \sigma}\left(c_{j, \sigma}^{\dagger} c_{j+1, \sigma}+\text { H.c. }\right)\right. \\
& -\frac{t_{0}^{2}}{U} \sum_{j} \hat{b}_{j, j+1}^{\dagger} \hat{b}_{j, j+1} \\
& \left.-\mathcal{J}_{0}\left(\frac{2 V}{\Omega}\right) \frac{t_{0}^{2}}{2 U}\left(\hat{b}_{j, j+1}^{\dagger} \hat{b}_{j+1, j+2}+\text { H.c. }\right)\right] \hat{P} .
\end{aligned}
$$

We now identify this as the Hamiltonian in Eq. (1), with $t=\mathcal{J}_{0}(V / \Omega) t_{0}, J=t_{0}^{2} / U$, and $\alpha=\mathcal{J}_{0}(2 V / \Omega)$. In an optical lattice context, where one has a fine degree of control over all parameters $t_{0}, U, \Omega$, and $V$, one can semi-independently vary $t, J$, and $\alpha$, and explore experimentally the effect of pair hopping on superconductivity in higher dimensions.
We note that this procedure is valid only when there is no "beating" between the oscillations at frequencies $\Omega$ and $U$. In other words, we require $|U-\Omega| \gg t$. The method can be generalized to the near-resonant case by simultaneously eliminating the driving term along with an amount $\Omega$ of the interaction term, leaving a Hamiltonian with an effective on-site repulsion $U-\Omega$, as discussed in [15,21].

\section{APPENDIX B: DETAILS OF THE DMRG CALCULATION}

In this section, we summarize some technical details of the DMRG calculation. The advantage of using a finite-size algorithm rather than infinite DMRG is that we may use symmetries to exactly fix the number of fermions in the system $N_{\uparrow}$ and $N_{\downarrow}$, which allows the precise determination of quantities such as the spin gap and compressibility.

The drawback of studying such a finite system with open boundaries is that it requires us to consider the interplay between finite-size and finite-entanglement scaling [49]. As we detail in the following, we find that our results are dominated by finite-size effects rather than finite-entanglement artifacts, and so we extrapolate only to $L \rightarrow \infty$ for the largest value of $\chi$ used.

We show some typical finite-size extrapolations in Figs. 7(a) and 7(b). In Fig. 7(a), we show the spin gap at $\alpha=0.5$, $n=7 / 32$ as a function of inverse system size $1 / L$ for various values of $J / t$. The solid lines are quadratic fits for different interaction strengths. The crosses are data points for different system sizes and values of $\chi=100-500$ (increasing $\chi$ makes almost no difference in the results and thus the multiple crosses appear as a single cross). Similarly, we show the extrapolation of the Luttinger parameter in Fig. 7(b), where the lines and 
crosses have the same meaning as in Fig. 7(a). In Figs. 7(d) and $7(\mathrm{e})$, we repeat these plots for the system parameters $\alpha=0.15$, $n=1 / 2$.

In Fig. 7(c), we show the nearest-neighbor singlet correlation function at various system sizes for the same parameters as in plots 7(a) and 7(b), while Fig. 7(f) shows the singlet correlations for the same parameters as in plots 7(d) and 7(e), with $\chi=300$. We see a polynomial decay over a significant range of distances $r$, after which an exponential tail develops. Such exponential tails always appear due to finite size and finite entanglement in some combination. The value of $r$ at which this exponential tail sets in increases as we increase the system size. Furthermore, at these bond dimensions, the typical truncation error is small $\left(\sim 10^{-4}\right)$. We conclude that at these bond dimensions, finite-size effects dominate over finite-entanglement effects, and thus we extrapolate only in $L$ and not $\chi[49]$.

In all phase diagrams in this paper, we compute the ground state at intervals of $\Delta J=0.1$, and $\Delta n=1 / 16$. For each quantity which determines a phase boundary, a linear interpolation is performed at the boundary of these grid squares to obtain a set of approximate grid points for the phase boundary. We then interpolate these points with a smoothed cubic spline to produce the plotted contour lines.
[1] F. C. Zhang and T. M. Rice, Effective Hamiltonian for the superconducting $\mathrm{Cu}$ oxides, Phys. Rev. B 37, 3759 (1988).

[2] E. Dagotto, Correlated electrons in high-temperature superconductors, Rev. Mod. Phys. 66, 763 (1994).

[3] P. W. Anderson, The resonating valence bond state in $\mathrm{La} 2 \mathrm{CuO} 4$ and superconductivity, Science 235, 1196 (1987).

[4] P. A. Lee, N. Nagaosa, and X. G. Wen, Doping a Mott insulator: Physics of high-temperature superconductivity, Rev. Mod. Phys. 78, 17 (2006).

[5] E. Demler, W. Hanke, and S. C. Zhang, $S O(5)$ theory of antiferromagnetism and superconductivity, Rev. Mod. Phys. 76, 909 (2004).

[6] K. A. Chao, J. Spalek, and A. M. Oles, Kinetic exchange interaction in a narrow s-band, J. Phys. C: Solid State Phys. 10, L271 (1977).

[7] F. H. L. Essler, H. Frahm, F. Gohmann, A. Klumper, and V. E. Korepin, The One-dimensional Hubbard Model (Cambridge University Press, Cambridge, 2005).

[8] S. Kaiser, S. R. Clark, D. Nicoletti, G. Cotugno, R. I. Tobey, N. Dean, S. Lupi, H. Okamoto, T. Hasegawa, D. Jaksch, and A. Cavalleri, Optical properties of a vibrationally modulated solid state Mott insulator, Sci. Rep. 4, 3823 EP (2014).

[9] R. Singla, G. Cotugno, S. Kaiser, M. Först, M. Mitrano, H. Y. Liu, A. Cartella, C. Manzoni, H. Okamoto, T. Hasegawa, S. R. Clark, D. Jaksch, and A. Cavalleri, THz-Frequency Modulation of the Hubbard $U$ in an Organic Mott Insulator, Phys. Rev. Lett. 115, 187401 (2015).

[10] R. Mankowsky, M. Först, and A. Cavalleri, Non-equilibrium control of complex solids by nonlinear phononics, Rep. Prog. Phys. 79, 064503 (2016).

[11] D. Nicoletti and A. Cavalleri, Nonlinear light-matter interaction at terahertz frequencies, Adv. Opt. Photon. 8, 401 (2016).

[12] J. Mentink, K. Balzer, and M. Eckstein, Ultrafast and reversible control of the exchange interaction in Mott insulators, Nat. Commun. 6, 6708 (2015).

[13] M. Bukov, L. D’ Alessio, and A. Polkovnikov, Universal highfrequency behavior of periodically driven systems: From dynamical stabilization to Floquet engineering, Adv. Phys. 64, 139 (2015).

[14] F. Meinert, M. J. Mark, K. Lauber, A. J. Daley, and H.-C. Nägerl, Floquet Engineering of Correlated Tunneling in the Bose-Hubbard Model with Ultracold Atoms, Phys. Rev. Lett. 116, 205301 (2016).
[15] M. Bukov, M. Kolodrubetz, and A. Polkovnikov, SchriefferWolff Transformation for Periodically Driven Systems: Strongly Correlated Systems with Artificial Gauge Fields, Phys. Rev. Lett. 116, 125301 (2016).

[16] J. J. Mendoza-Arenas, F. J. Gómez-Ruiz, M. Eckstein, D. Jaksch, and S. R. Clark, Ultra-fast control of mangetic relaxation in a periodically driven Hubbard model, Ann. Phys. 529, 1700024 (2017).

[17] J. R. Coulthard, S. R. Clark, S. Al-Assam, A. Cavalleri, and D. Jaksch, Enhancement of superexchange pairing in the periodically driven Hubbard model, Phys. Rev. B 96, 085104 (2017).

[18] R. A. Hart, P. M. Duarte, T. L. Yang, X. Liu, T. Paiva, E. Khatami, R. T. Scalettar, N. Trivedi, D. A. Huse, and R. G. Hulet, Observation of antiferromagnetic correlations in the hubbard model with ultracold atoms, Nature (London) 519, 211 (2015).

[19] T. A. Hilker, G. Salomon, F. Grusdt, A. Omran, M. Boll, E. Demler, I. Bloch, and C. Gross, Revealing hidden antiferromagnetic correlations in doped Hubbard chains via string correlators, Science 357, 484 (2017).

[20] H. Lignier, C. Sias, D. Ciampini, Y. Singh, A. Zenesini, O. Morsch, and E. Arimondo, Dynamical Control of Matter-Wave Tunneling in Periodic Potentials, Phys. Rev. Lett. 99, 220403 (2007).

[21] R. Desbuquois, M. Messer, F. Görg, K. Sandholzer, G. Jotzu, and T. Esslinger, Controlling the Floquet state population and observing micromotion in a periodically driven two-body quantum system, Phys. Rev. A 96, 053602 (2017).

[22] F. Görg, M. Messer, K. Sandholzer, G. Jotzu, R. Desbuquois, and T. Esslinger, Enhancement and sign change of magnetic correlations in a driven quantum many-body system, Nature (London) 553, 481 (2018).

[23] A. Moreno, A. Muramatsu, and S. R. Manmana, Ground-state phase diagram of the one-dimensional $t$ - $J$ model, Phys. Rev. B 83, 205113 (2011).

[24] S. R. White, Density Matrix Formulation for Quantum Renormalization Groups, Phys. Rev. Lett. 69, 2863 (1992).

[25] U. Schollwöck, The density-matrix renormalization group in the age of matrix product states, Ann. Phys. (NY) 326, 96 (2011).

[26] M. Ogata, M. U. Luchini, S. Sorella, and F. F. Assaad, Phase Diagram of the One-Dimensional $t$ - $J$ Model, Phys. Rev. Lett. 66, 2388 (1991).

[27] K. Sano, Possible phase diagram of a $t$ - $J$ ladder model, J. Phys. Soc. Jpn. 65, 1146 (1996). 
[28] P. Corboz, T. M. Rice, and M. Troyer, Competing States in the $t$ - $J$ Model: Uniform $d$-Wave State Versus Stripe State, Phys. Rev. Lett. 113, 046402 (2014).

[29] H. Eskes and A. M. Oleś, Two Hubbard bands: Weight Transfer in Optical and One-Particle Spectra, Phys. Rev. Lett. 73, 1279 (1994).

[30] H. Eskes, A. M. Oleś, M. B. J. Meinders, and W. Stephan, Spectral properties of the Hubbard bands, Phys. Rev. B 50, 17980 (1994).

[31] J. Spałek, Effect of pair hopping and magnitude of intra-atomic interaction on exchange-mediated superconductivity, Phys. Rev. B 37, 533 (1988).

[32] B. Ammon, M. Troyer, and H. Tsunetsugu, Effect of the threesite hopping term on the $t-J$ model, Phys. Rev. B 52, 629 (1995).

[33] E. Ercolessi, P. Pieri, and M. Roncaglia, Phase separation and three-site hopping in the two-dimensional $t$ - $J$ model, Phys. Lett. A 233, 451 (1997).

[34] Y. Saiga and M. Imada, Competition between spin exchange and correlated hopping, J. Phys. Chem. Solids 63, 1531 (2002).

[35] J. Jedrak and J. Spalek, Renormalized mean-field $t$ - $J$ model of high- $T_{c}$ superconductivity: Comparison to experiment, Phys. Rev. B 83, 104512 (2011).

[36] S. Al-Assam, S. R. Clark, and D. Jaksch, The tensor network theory library, J. Stat. Mech.: Theor. Exp. (2017) 093102.

[37] V. J. Emery, S. A. Kivelson, and H. Q. Lin, Phase Separation in the $t$ - $J$ Model, Phys. Rev. Lett. 64, 475 (1990).

[38] In our results, the fermions tend to cluster on the left because the DMRG algorithm always finishes with a leftward minimization sweep, breaking the reflection symmetry of the system.
[39] S. Ejima, F. Gebhard, and S. Nishimoto, Tomonaga-Luttinger parameters for doped Mott insulators, Europhys. Lett. 70, 492 (2005).

[40] T. Giamarchi, Quantum Physics in One Dimension (Oxford University Press, Oxford, 2004).

[41] D. S. Rokhsar and S. A. Kivelson, Superconductivity and the Quantum Hard-Core Dimer Gas, Phys. Rev. Lett. 61, 2376 (1988).

[42] M. Rigol and A. Muramatsu, Ground-state properties of hardcore bosons confined on one-dimensional optical lattices, Phys. Rev. A 72, 013604 (2005).

[43] S. Ejima, H. Fehske, and F. Gebhard, Dynamic properties of the one-dimensional Bose-Hubbard model, Europhys. Lett. 93, 30002 (2011).

[44] Michele Dolfi, Bela Bauer, Matthias Troyer, and Zoran Ristivojevic, Multigrid Algorithms for Tensor Network States, Phys Rev. Lett. 109, 020604 (2012).

[45] M. Vojta, A. Hübsch, and R. M. Noack, Phase diagram of the quarter-filled extended Hubbard model on a two-leg ladder, Phys. Rev. B 63, 045105 (2001).

[46] J. H. Shirley, Solution of the Schrödinger equation with a Hamiltonian periodic in time, Phys. Rev. 138, B979 (1965).

[47] F. Casas, J. A. Oteo, and J. Ros, Floquet theory: Exponential perturbative treatment, J. Phys. A: Math. Gen. 34, 3379 (2001).

[48] A. Eckardt and E. Anisimovas, High-frequency approximation for periodically driven quantum systems from a Floquet-space perspective, New J. Phys. 17, 093039 (2015).

[49] M. Dolfi, B. Bauer, S. Keller, and M. Troyer, Pair correlations in doped Hubbard ladders, Phys. Rev. B 92, 195139 (2015). 